\title{
Balance Studies on Compartmental Systems with Stochastic Inputs
}

\author{
JOHN A. JACQUEZ \\ Department of Biostatistics and Department of Physiology, \\ The University of Michigan, Ann Arbor, Michigan, U.S.A.
}

AND

Frances J. MATHER

Department of Biostatistics, The University of Michigan, Ann Arbor, Michigan, U.S.A.

(Received 23 August 1965, and in revised form 25 February 1966)

For a population of identical one compartment systems with daily inputs which are random samples from a stationary distribution the mean of the daily balance (balance $=$ input minus excretion) taken over the population of compartments or over days for one compartment is linearly dependent on input with a slope which is independent of the distribution of the inputs and an intercept which depends only on the mean of the distribution of intakes. Analytical and simulation studies on a model with three daily inputs gives essentially similar results. Even if the inputs are not from a stationary distribution but the inputs to a population of compartments are all from the same distribution, one still obtains a linear regression of the mean of the daily balance over the population of compartments, on intake for any particular day but this no longer holds from day to day. This provides a theoretical basis for the interpretation of balance studies on populations of individuals when the daily intakes of an element under study cannot bc kcpt constant.

\section{Introduction}

The clinical investigator is frequently interested in measuring the intake and excretion of various dietary constituents and the balance between intake and excretion. Under ideal conditions, such a "balance study" is carried out by putting the patient on a constant dietary intake and measuring both intake and excretion. However, it must be emphasized that it is often not possible to maintain a constant intake over a long study period. Such studies are useful in defining the nutritional needs for normal function as well as many abnormalities of absorption, metabolism or excretion in disease states. 
A balance study is an application of the law of conservation of mass to an open system. At any time, the rate at which an element enters the system minus the rate at which it leaves the system must equal the rate of accumulation of the element in the system. For many elements the rate of excretion from living systems is proportional to the amount in some compartment or compartments. For example, the assumption that urinary excretion of inorganic iodine is proportional to the amount in the extracellular space is a good assumption, if there are no large changes in renal function during the course of an experiment. If the intake is constant, such systems approach a stationary state in which the daily excretion equals the daily intake. If the intake is then changed to a new level, the system goes through a transition period which lasts for a few turnover times of the element in the system, after which the system is in a new stationary state. However, in studies on populations in the natural state considerable fluctuation is found in the daily intake of many elements. Rustagi (1964) has shown that the distribution of daily intakes of lead, cadmium and chromium approximates the log-normal distribution. The daily intake of iodine in humans also follows a skewed distribution which approximates a log-normal distribution (Vought \& London, 1964a). For such circumstances, it is not obvious how the daily balances, that is the balances calculated from each day's intake and excretion, depend on the distribution of intakes and on the turnover time of the system for the particular element under study. This problem was first brought to our attention when we attempted to analyze a set of data on daily balances of iodine from a study of iodine balance on a group of pregnant women (Dworkin, Jacquez \& Beierwaltes, unpublished data).

The present paper is an attempt to answer some of these questions. For this purpose we will examine some simple one-compartment models with use of the assumptions that (i) the rate of excretion is directly proportional to the quantity of the element in the system, and (ii) the intake is a random sample from a stationary distribution.

\section{A Simplified Model with One Intake Per Day}

To bring out the basic features of the problem lct us first consider a uniformly and instantaneously mixed one compartment system for which the intake occurs as a single instantaneous input at the same time each day. We develop a finite difference model using a basic time unit of one day. Let us introduce the following notation and assumptions:

$x_{i}$ The amount ingested on day $i$. It is assumed that this is a random sample from a stationary distribution. For this model we make the further simplifying assumption that this intake is instantaneous and occurs at the start of day $i$. 
$q_{l}$ The total amount of the element in the compartment at the start of day $i$, just before the intake $x_{i}$.

$k$ The excretion constant. The excretion rate at any time is assumed to equal $k$ times the amount in the compartment.

Thus the amount present in the compartment at the start of day $i$ is $q_{i}+x_{i}$ and the amount excreted on the $i$ th day is $\left(q_{i}+x_{i}\right)\left(1-\mathrm{e}^{-k}\right)$. Note that the turnover time for the element in question is $1 / k$ days.

The amount of element remaining in the system at the end of day $i$ is $q_{i+1}$.

$$
q_{i+1}=\left(q_{i}+x_{i}\right) \mathrm{e}^{-k} \text {. }
$$

Suppose that this process has been going on for $n$ days and that the amount in the compartment at the start of the process was $q_{1}$. Substituting the corresponding equation for $q_{i}, q_{i-1}, \ldots$ into equation (1) gives equation (2) for $q_{i+1}$.

$$
q_{i+1}=x_{i} \mathrm{e}^{-k}+x_{i-1} \mathrm{e}^{-2 k}+x_{i-2} \mathrm{e}^{-3 k}+\ldots+x_{i-n-1} \mathrm{e}^{-n k}+q_{1} \mathrm{e}^{-n k} .
$$

From the assumption that $x_{j}$ is a random sample from a stationary distribution, we have $E\left(x_{i}\right)=E\left(x_{i-1}\right)=\ldots E\left(x_{i-n-1}\right)=\mu_{x}$ where $E()$ is the expected value operator. (In the remainder of the paper we use the term "expected value" in its technical sense in statistics. If $\varphi(x)$ is a distribution function and $g(x)$ is any function of the random variable $x$, the expected value of $g(x)$ is, $E[g(x)]=\int g(x) \varphi(x) \mathrm{d} x$.) Taking expected values of both sides of equation (2) and using the notation $E\left(q_{i+1}\right)=\mu_{q i+1}$, gives

$$
\mu_{q_{i+1}}=\mu_{x} \mathrm{e}^{-k} \sum_{0}^{n-1} \mathrm{e}^{-r k}+E\left(q_{1}\right) \mathrm{e}^{-n k} \text {. }
$$

The finite sum in equation (3) is a truncated power series which may be summed to give equation (4)

$$
\mu_{q_{i+1}}=\mu_{x} \mathrm{e}^{-k} \frac{\left(1-\mathrm{e}^{-n k}\right)}{1-\mathrm{e}^{-k}}+E\left(q_{1}\right) \mathrm{e}^{-n k} .
$$

If $n$ is large and $E\left(q_{1}\right)$ is of the same order of magnitude as $\mu_{x}$, the terms in $\mathrm{e}^{-n k}$ are very small and may be neglected. We note that this is not a very stringent requirement, thus if $\mathrm{e}^{-k}=0.5, \mathrm{e}^{-10 k}<0.001$ and the above assumption is adequate for $n>10$. This assumption is equivalent to writing equation (2) as an infinite series, as though the process had been going on for an infinitely long time; we use it without further comment in the remainder of the paper. With this assumption $E\left(q_{i}\right)=E\left(q_{i+1}\right)=\ldots=\mu_{q}$, and equation (4) may be written as in equation (5).

$$
\mu_{q}=\mu_{x} \frac{\mathrm{e}^{-k}}{1-\mathrm{e}^{-k}} \text {. }
$$


By definition, the balance for day $i, b_{i}$, is the intake minus the excretion, equation (6).

$$
b_{i}=x_{i}-q_{i}\left(1-e^{-k}\right)-x_{i}\left(1-e^{-k}\right)=x_{i} \mathrm{e}^{-k}-q_{i}\left(1-\mathrm{e}^{-k}\right) .
$$

We are interested in finding the conditional expected value of $b_{i}$ for given intake $x_{i}$, that is, the expected value of $b_{i}$ for $x_{i}$ fixed, $\mu_{t_{i}}$. This is obtained by taking expected values of both sides of equation (6) but treating $x_{i}$ as if it were a constant.

$$
\mu_{b_{i}}=x_{i} \mathrm{e}^{-k}-\mu_{q}\left(1-\mathrm{e}^{-k}\right)=\left(x_{i}-\mu_{x}\right) \mathrm{e}^{-k} .
$$

Thus the expected daily balance is a linear function of the intake. In other words, equation (7) predicts a linear regression of daily balances on the intakes with a slope, $\mathrm{e}^{-k}$, which is a function of the turnover time only, and an intercept of $-\mu_{x} \mathrm{e}^{-k}$. Note that this system has the property of ergodicity; that is, given a population of identical individuals, the means in equation (7) can be interpreted as means over the population on any one day or as means over days for any one member of the population; for either case equation (7) gives the regression of mean balance on the intake. Furthermore, the parameters of the regression line depend only on the mean value of the distribution of intakes but are otherwise independent of the distribution of intakes. The main property of the distribution of intakes which has been essential to the argument so far is the assumption of stationarity. If $\sigma_{x}^{2}$ is the variance of the distribution of intakes, it is not difficult to show that the variance around the regression line, $\sigma_{b}^{2}=E\left[\left(b_{i}-\mu_{b_{1}}\right)^{2}\right]$, is given by equation (8).

$$
\sigma_{b}^{2}=E\left[\left(b_{i}-\mu_{b_{1}}\right)^{2}\right]=\frac{\mathrm{e}^{-2 k}\left(1-\mathrm{e}^{-k}\right)^{2}}{1-\mathrm{e}^{-2 k}} \sigma_{x}^{2}
$$

\section{A Population of Compartments}

Retaining the previous simplified model, suppose we have a population of individuals (compartments) which is characterized by some distribution of values of $k, k_{j}$ being the excretion constant for the $j$ th individual. Let $x_{i j}$ be the amount taken in by the $j$ th individual on the $i$ th day and let $q_{i j}$ be the total amount in the $j$ th individual at the start of the $i$ th day. We further assume that the distribution of intakes is stationary and that the distributions of intakes and of the $k_{j}$ are independent. Equation (1) then becomes equation (9).

$$
q_{i+1, j}=\left(q_{i j}+x_{i j}\right) \mathrm{e}^{-k_{j}} \text {. }
$$

Equation (2) also is modified slightly (equation (10)).

$$
q_{i+1, j}=x_{i j} \mathrm{e}^{-k_{j}}+x_{i-1, j} \mathrm{e}^{-2 k_{j}}+\ldots
$$


Taking expected values over the distribution of intakes, we obtain equation (11) for the $j$ th individual.

$$
\mu_{q j}=\mu_{x} \mathrm{e}^{-k_{j}} \sum \mathrm{e}^{-r k_{j}}=\frac{\mu_{x} \mathrm{e}^{-k_{j}}}{1-\mathrm{e}^{-k_{j}}} .
$$

The balance for the $j$ th individual on the $i$ th day is then equation (12)

$$
b_{i j}=x_{i j} \mathrm{e}^{-k_{j}}-q_{i j}\left(1-e^{-k_{j}}\right) .
$$

For any one individual, the results are the same as before. Now in equations (11) and (12) take expected values over the distribution of the $k_{j}$ to obtain equations (13) and (14).

$$
\begin{aligned}
& \mu_{q}=\mu_{x} E\left[\frac{\mathrm{e}^{-k_{j}}}{1-\mathrm{e}^{-k_{j}}}\right] \\
& \mu_{b i}=\left(x_{i}-\mu_{x}\right) E\left[\mathrm{e}^{-k_{j}}\right] .
\end{aligned}
$$

The effect of the distribution of the $k_{j}$ may be seen by expanding the arguments of the expected value operator in Taylor's series around the mean $\mu_{k}$ and then carrying out the expected value operations term by term. For the balances (equation (14)) this gives the simple expression, equation (15).

$$
\mu_{b i}=\left(x_{i}-\mu_{x}\right) \mathrm{e}^{-\mu_{k}}\left[1+\frac{\sigma_{k}^{2}}{2 !}-\frac{\mu_{k 3}}{3 !}+\frac{\mu_{k 4}}{4 !} \ldots\right] \text {. }
$$

In this equation $\mu_{k 3}$ and $\mu_{k 4}$ are the third and fourth moments around the population mean, $\mu_{k}$, and $\sigma_{k}^{2}$ is the variance. Again a linear regression is obtained but the slope depends on the moments of the distribution of the $k_{j}$.

\section{Distributed Inputs}

Now let us consider how the results of the previous model are changed if the intake in one day is distributed over the course of the day. For the general case, if the intake in time $\mathrm{d} t$ at $t$ is $x_{1}(t) \mathrm{d} t$, the amount of the ingested element remaining at the end of day $i$ is:

$$
\int_{0}^{1} x_{i}(t) \mathrm{e}^{-(1-t) k} \mathrm{~d} t
$$

Then the total remaining in the system at the end of the day is given by equation (16).

$$
q_{t+1}=q_{i} \mathrm{e}^{-k}+\int_{0}^{1} x_{i}(t) \mathrm{e}^{-(1-t) k} \mathrm{~d} t
$$

For practical purposes, the major intake of any element occurs at three distinct meals, so we consider a model with three inputs. Suppose the intakes on day $i$ are $x_{i 1}, x_{i 2}$ and $x_{13}$ at times $t_{1}, t_{2}$ and $t_{3}$, respectively. Assume that each of the intakes is a random sample from a stationary distribution. The 
times of ingestion, $t_{1}, t_{2}$ and $t_{3}$ may also be considered to be random variables but we do not do this here. Then the amount present at the end of day $i$ is given by equation (17).

$$
q_{i+1}=q_{i} \mathrm{e}^{-k}+x_{i 1} \mathrm{e}^{-k\left(1-t_{1}\right)}+x_{i 2} \mathrm{e}^{-k\left(1-t_{2}\right)}+x_{i 3} \mathrm{e}^{-k\left(1-t_{3}\right)} .
$$

Although the development can now be carried out as before, this approach turns out not to be useful because in most balance studies only the total intake in a day is measured. This suggests that we attempt to reformulate the problem in terms of the total intake, $x_{i}=x_{i 1}+x_{i 2}+x_{i 3}$. Referring back to equation (10) let us define $\lambda_{i}$ so that equation (18) holds.

$$
x_{i 1} \mathrm{e}^{-k\left(1-t_{1}\right)}+x_{i 2} \mathrm{e}^{-k\left(1-t_{2}\right)}+x_{i 3} \mathrm{e}^{-k\left(1-t_{3}\right)}=x_{i} \mathrm{e}^{-\lambda_{1}} .
$$

Given $x_{i 1}, x_{i 2}, x_{i 3}$ and $t_{1}, t_{2}$ and $t_{3}$, equation (18) can be solved for $\lambda_{1}$. Thus we replace the original problem with one in which all of the intake is assumed to occur at the start of the day but now the excretion constant for the input $x_{i}$ is a stochastic variable dependent on $x_{i j}$ and $t_{j}$. The amount remaining in the compartment at the end of day $i$ is now given by equation (19)

$$
q_{i+1}=q_{i} \mathrm{e}^{-k}+x_{i} \mathrm{e}^{-\lambda_{i}} .
$$

It is convenient at this point to change to the variate $K_{i}=\mathrm{e}^{-\lambda_{i}}$, equation (20).

$$
q_{i+1}=q_{i} \mathrm{e}^{-k}+K_{i} x_{i} \text {. }
$$

Equation (20) can again be expanded into an infinite series.

$$
q_{i+1}=K_{i} x_{i}+K_{i-1} x_{i-1} \mathrm{e}^{-k}+K_{i-2} x_{i-2} \mathrm{e}^{-2 k}+\ldots
$$

Taking expected values and noting that $E\left[K_{j} x_{j}\right]=\mu_{K} \mu_{x}+\sigma_{K x}$, where $\sigma_{K x}$ is the covariance of $K$ and $x$, leads to equation (22)

$$
\mu_{q}=\left[\mu_{K} \mu_{x}+\sigma_{K x}\right] \sum_{n=0}^{\infty} \mathrm{e}^{-n k}=\frac{\mu_{K} \mu_{x}+\sigma_{K x}}{1-\mathrm{e}^{-k^{-2}} .}
$$

The balance for day $i$ is now given by equation (23).

$$
b_{i}=x_{i}-q_{i}\left[1-\mathrm{e}^{-k}\right]-x_{i}\left[1-K_{i}\right]=K_{i} x_{i}-q_{i}\left[1-\mathrm{e}^{-k}\right] .
$$

The conditional expected value of $b_{i}$, for given intake $x_{i}$, is then

$$
\mu_{b_{i}}=x_{i} E\left[K_{i} / x_{i}\right]-\mu_{K} \mu_{x}-\sigma_{K x}
$$

where $E\left[K_{i} / x_{i}\right]$ is the conditional mean of $K_{i}$ given that $x$ equals $x_{i}$. Assume that $E\left[K_{i} / x_{i}\right]$ depends linearly on $x_{i}$. Thus we consider only a linear regression of $K_{i}$ on $x_{i}$. Then $E\left[K_{i} / x_{i}\right]$ is given by equation (25).

$$
E\left[K_{i} / x_{i}\right]=\mu_{K}+\frac{\sigma_{K x}}{\sigma_{x}^{2}}\left(x_{i}-\mu_{x}\right) .
$$

Finally, substituting equation (25) into (24) gives equation (26) for $\mu_{b_{i}}$

$$
\mu_{b_{l}}=\left(\mu_{K}+\frac{\sigma_{K x}}{\sigma_{x}^{2}} \mu_{x}\right)\left(x_{i}-\mu_{x}\right)-\sigma_{K x}+\frac{\sigma_{K x}}{\sigma_{x}^{2}}\left(x_{i}-\mu_{x}\right)^{2} .
$$


In this case, $\mu_{b_{i}}$ contains a quadratic term in $x_{i}$. To estimate the relative contributions of the linear and quadratic terms note that for $x_{i}-\mu_{x}=\sigma_{x}$, the quadratic term becomes $\sigma_{K x}$ whereas the linear terms reduce to $\sigma_{x} \mu_{K}+\sigma_{K x} \mu_{x} / \sigma_{x}-\sigma_{K x}$. For many cases of interest $\mu_{x} \gg \sigma_{x}$ and $\mu_{K}>\sigma_{K x}$ and the quadratic term becomes small in comparison to the linear term. The problem simulated in the next section provides a good example of such a case.

\section{Simulation of a One Compartment Model with Three Daily Stochastic Inputs}

In order to compare the predictions of this theory with some reported results on iodine balance, we first simulated a one compartment model with stochastic inputs at three fixed times on each day. The times chosen were $0.02,0.17$ and 0.42 days. The mean values and standard deviations were chosen to correspond approximately with the data reported by Vought \& London on iodine intakes at breakfast, lunch and dinner (Vought \& London, $1964 b$ ). The program was written in MAD (Arden, 1963; Galler, 1962) and compiled and run on the IBM 7090 at The University of Michigan Computing Center. Starting with an amount $q_{i}$ in the compartment on day $i$ the logarithms of each of the three intakes for day $i$ were chosen by drawing random samples from three normal distributions which had means of $3 \cdot 2,4 \cdot 3$ and 4.3 and standard deviations $0.45,0.60$ and 0.60 , respectively. An excretion constant of $k=0.6$ was used. The total intake $x_{i}$, the amount excreted, the balance $b_{i}$ and the values of $K_{i}$ and $\lambda_{i}$ were then calculated and finally

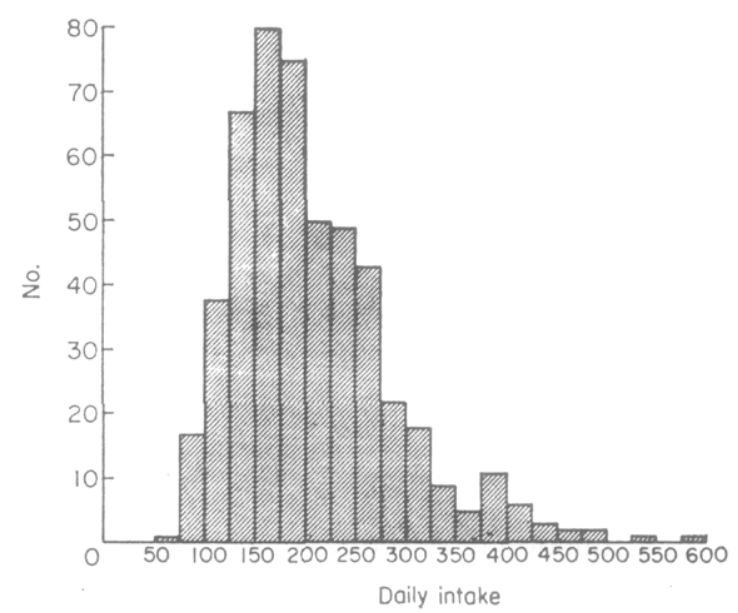

FIG. 1. Histogram of distribution of daily intakes on sample of 500 . 
the amount remaining at the end of day $i, q_{i+1}$, was calculated. This process was then repeated for days $i+1, i+2, \ldots$. To initiate the calculations, $q_{1}$ was chosen close to the mean value $\mu_{q}$. The simulation was run for 500 consecutive days. The distributions of the $x_{i}, K_{i}$ and $b_{i}$, which were obtained are shown in Figs 1 to 3 and the mean values, standard deviations and covariances calculated from the sample of 500 are given in Table 1. For

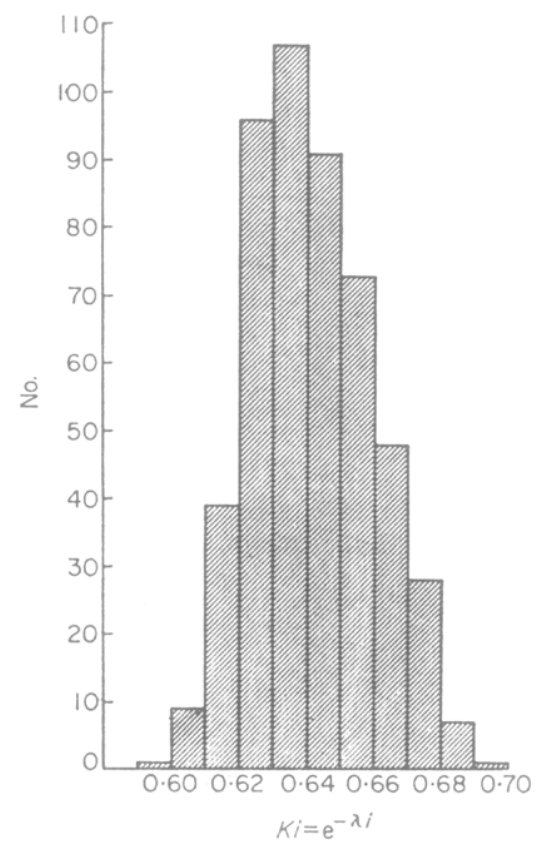

FIG. 2. Histogram of distribution of $K_{t}=\mathrm{e}^{-\lambda_{t}} ; \lambda_{t}$ is the apparent excretion constant for the ingested material on the assumption it is all taken in at the start of the day.

TABLE 1

Results of simulation on one compartment model with stochastic inputs

\begin{tabular}{cccc}
\hline Variable & $\begin{array}{c}\text { Sample } \\
\text { mean }\end{array}$ & $\begin{array}{c}\text { Sample } \\
\text { standard deviation }\end{array}$ & $\begin{array}{c}\text { Covariance } \\
\text { with } x\end{array}$ \\
\hline$x_{1}$ & 26.7 & $13 \cdot 4$ & \\
$x_{2}$ & $93 \cdot 0$ & 60.6 & \\
$x_{3}$ & $87 \cdot 2$ & $54 \cdot 6$ & \\
$x$ & 207.0 & $79 \cdot 9$ & \\
$q$ & $295 \cdot 3$ & $63 \cdot 9$ & -0.248 \\
$\lambda$ & $0 \cdot 444$ & $0 \cdot 028$ & 0.165 \\
$K=\mathrm{e}^{-\lambda}$ & 0.642 & $0 \cdot 018$ & 4021 \\
$b$ & -0.263 & $58 \cdot 1$ & \\
\hline
\end{tabular}




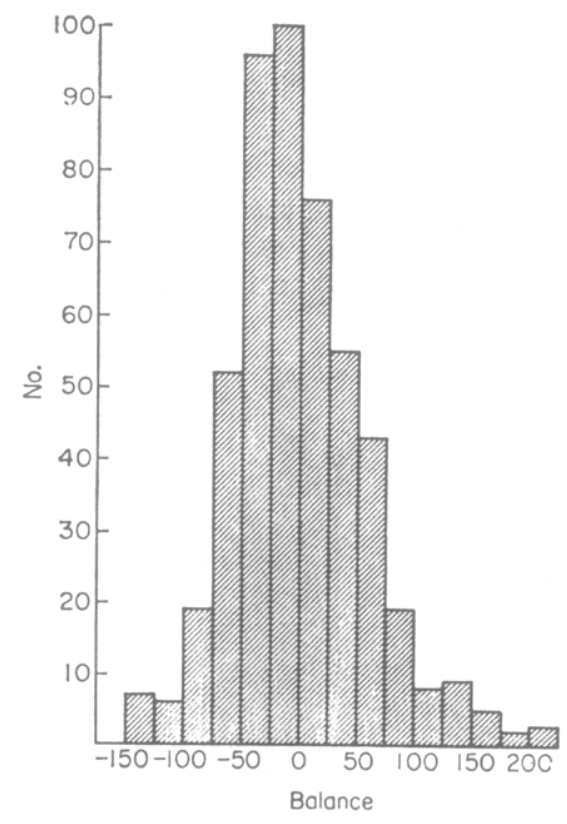

Fig. 3. Histogram of distribution of balances obtained on sample of 500 on the one compartment model with three stochastic intakes.

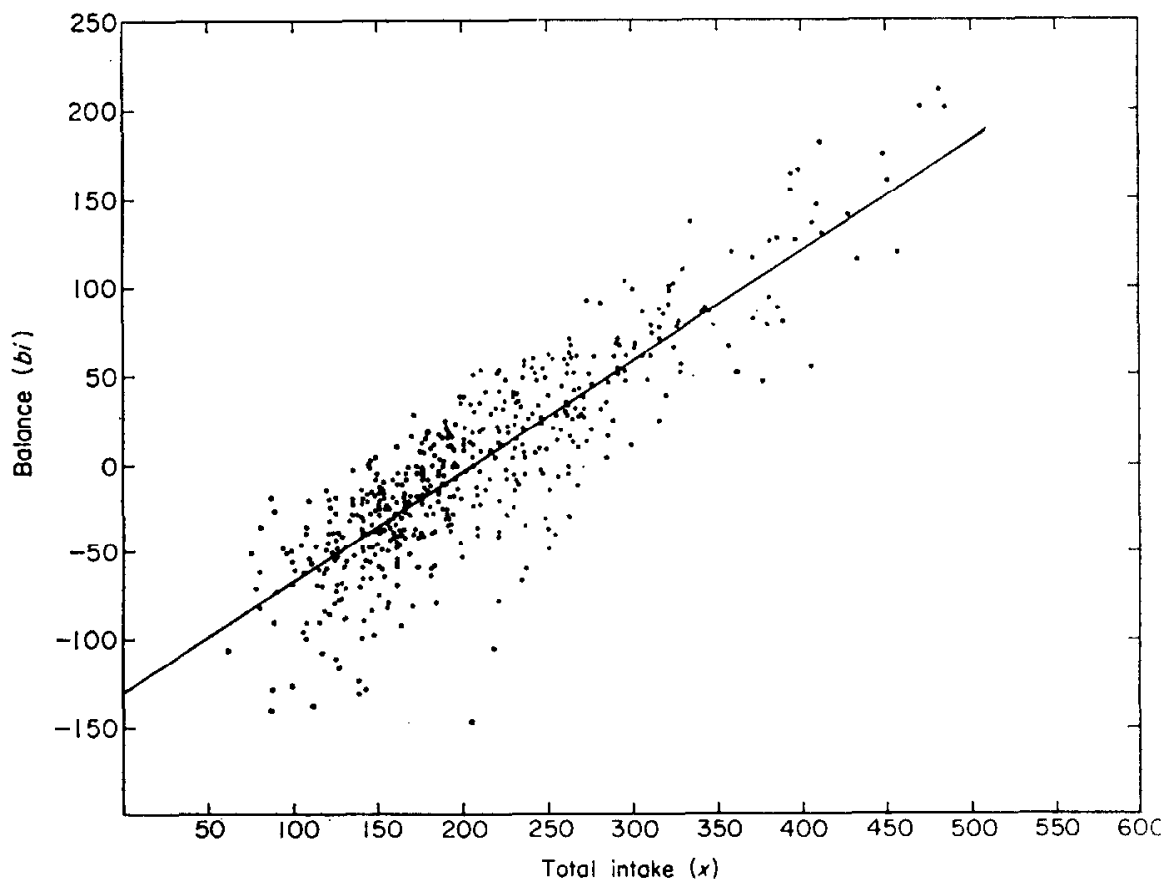

Fio. 4. Scattergram showing dependence of balances on intake for the 500 samples drawn from the model. 
comparison with data on iodine balance (Vought \& London, 1964a; Vought \& London, 1964b), the intake and balance may be given the units of micrograms of iodine per day. Figure 4 shows a scattergram of the balances and the best fitting linear regression line. The intercept and slope of the regression line are -130.6 and 0.6296 with standard deviations of 3.6 and 0.016 , respectively. The calculation of the parameters $\mu_{K}, \sigma_{K x}$ of the bivariate distribution of the $K_{i}$ and $x_{i}$ is a difficult task which we have not attacked. However, if we use the sample means and covariances given in Table 1 as approximations to the parameter values and substitute these in equation (26), neglecting the quadratic term which is quite small, the intercept and slope predicted by equation (26) are -132.9 and 0.636 , respectively.

\section{The Non-stationary State}

The assumption of a stationary distribution of intakes has been essential to the developments so far. It is important to know to what extent this assumption may be relaxed. For example, we expect that the equations developed so far would change little if in a study on a population the parameters of the distribution of intakes changed slowly in comparison to the turnover time of the element under study. We consider the simplified model with one intake but now assume that the distribution function for the intake varies from day to day. First we note that equation (2) holds for all cases. However, $E\left[x_{i}\right]=\mu_{x}(i)$ where $\mu_{x}(i)$ is the mean of the distribution of intakes for the $i$ th day. Taking expected values, gives equation (27).

$$
\mu_{q}(i+1)=E\left[q_{i+1}\right]=\mathrm{e}^{-k} \sum_{r=0}^{\infty} \mu_{x}(i-r) \mathrm{e}^{-r k} .
$$

The balance for day $i$ is again given by equation (6). If we take expected values for given $x_{i}$

$$
\mu_{b}(i)=x_{i} \mathrm{e}^{-k}-E\left[q_{i}\left(1-\mathrm{e}^{-k}\right)\right] .
$$

Substituting from equation (27)

$$
\mu_{b}(i)=x_{i} \mathrm{e}^{-k}-\left(1-\mathrm{e}^{-k}\right) \mathrm{e}^{-k} \sum_{r=0}^{\infty} \mu_{x}(i-r-1) \mathrm{e}^{-r k} .
$$

Thus for a population of identical individuals, if the intake of each individual on day $i$ is a random sample from the same distribution then equation (29) again predicts a linear regression of the mean daily balance for day $i$ on the intake for day $i$ with slope $\mathrm{e}^{-k}$; however, the intercept now changes from day to day. Furthermore, the dependence of mean daily balance on daily intake for different days for one individual is no longer necessarily linear but is now determined by the functional dependence of $\mu_{x}(i)$ on $i$. 


\section{The Transition Period for a Step Change in the Distribution of Inputs}

As part of our simulation studies on a model which was set up to simulate an iodine balance study, we ran a simulation of the transition phase after a change from one distribution of intakes to another. At the same time this provided an example of a non-stationary process for which the daily balances should fit equation (29). For this study the model with three daily stochastic intakes was used. The intakes on any one day were again assumed to be at $0.02,0.17$ and 0.42 days and the excretion constant was $k=0.6$. The system was alternated between two 10-day periods, one on a low intake and one on a high intake. For the high intake period the distributions of the logarithms of the three intakes for a day were normal distributions with means of $3.9,4.6,4.6$ and standard deviations of $0.50,0.65$ and 0.65 , respectively. For the low intake period the corresponding means and standard deviations were $2 \cdot 7,3 \cdot 7,3 \cdot 7$ and $0 \cdot 35,0 \cdot 50,0 \cdot 50$, respectively. The simulation was run for 100 such 20 -day cycles. The mean daily intake was 107.5 for the

TABLE 2

Summary of simulation study of transition periods between high and low intakes

\begin{tabular}{|c|c|c|c|c|c|c|}
\hline \multirow{2}{*}{$\frac{\text { Day }}{1}$} & \multirow{2}{*}{$\begin{array}{c}\begin{array}{c}\text { Amount in } \\
\text { compartment } \\
\text { mean }\end{array} \\
402 \pm 10.4 \dagger\end{array}$} & $\begin{array}{l}\text { Mean } \\
\text { intake }\end{array}$ & \multicolumn{2}{|c|}{$\begin{array}{c}\text { Mean } \\
\text { balance }\end{array}$} & \multirow{2}{*}{$\begin{array}{c}\begin{array}{c}\text { Mean } \\
\lambda\end{array} \\
0.445\end{array}$} & \multirow{2}{*}{$\frac{\begin{array}{c}\text { Mean } \\
\mathrm{e}^{-\lambda}\end{array}}{0.641}$} \\
\hline & & $106 \pm 3 \cdot 2$ & -123 & $\pm 5 \cdot 2$ & & \\
\hline 2 & $299 \pm 6.0$ & $104 \pm 2.9$ & -68 & \pm 3.0 & 0.447 & 0.640 \\
\hline 3 & $231 \pm 4.1$ & $106 \pm 3 \cdot 1$ & -36 & \pm 2.5 & 0.445 & 0.641 \\
\hline 4 & $195 \pm 3 \cdot 3$ & $102 \pm 2 \cdot 9$ & -22 & \pm 2.5 & 0.444 & 0.641 \\
\hline 5 & $172 \pm 2 \cdot 6$ & $106 \pm 3 \cdot 2$ & -10 & \pm 2.5 & 0.446 & 0.641 \\
\hline 6 & $163 \pm 2 \cdot 3$ & $113 \pm 3.9$ & -6 & \pm 2.6 & 0.444 & 0.642 \\
\hline 7 & $162 \pm 3 \cdot 0$ & $110 \pm 3 \cdot 4$ & -2 & \pm 2.4 & 0.445 & 0.641 \\
\hline 8 & $160 \pm 3.0$ & $113 \pm 3.6$ & 0 & $\pm 2 \cdot 8$ & 0.443 & 0.642 \\
\hline 9 & $160 \pm 2 \cdot 6$ & $107 \pm 3 \cdot 1$ & -4 & \pm 2.5 & 0.446 & 0.640 \\
\hline 10 & $156 \pm 2 \cdot 2$ & $109 \pm 3.0$ & 0 & $\pm 2 \cdot 2$ & 0.444 & 0.641 \\
\hline 11 & $156 \pm 2 \cdot 3$ & $316 \pm 13 \cdot 4$ & 130 & \pm 8.5 & 0.459 & 0.632 \\
\hline 12 & $286 \pm 9.0$ & $327 \pm 13 \cdot 8$ & 81 & \pm 10.4 & 0.446 & 0.641 \\
\hline 13 & $367 \pm 10.0$ & $307 \pm 12.7$ & 30 & \pm 8.7 & 0.452 & 0.637 \\
\hline 14 & $397 \pm 10.4$ & $304 \pm 11.5$ & 15 & \pm 8.7 & 0.452 & 0.637 \\
\hline 15 & $411 \pm 9.7$ & $307 \pm 13 \cdot 1$ & 10 & $\pm 10 \cdot 1$ & 0.452 & 0.637 \\
\hline 16 & $422 \pm 9.6$ & $306 \pm 13 \cdot 6$ & 6 & \pm 9.8 & 0.447 & 0.640 \\
\hline 17 & $428 \pm 10 \cdot 7$ & $298 \pm 12 \cdot 2$ & -4 & \pm 9.7 & 0.455 & 0.635 \\
\hline 18 & $425 \pm 9.2$ & $293 \pm 11 \cdot 1$ & -6 & $\pm 8 \cdot 2$ & 0.455 & 0.634 \\
\hline 19 & $419 \pm 8.6$ & $305 \pm 13 \cdot 5$ & 6 & \pm 9.5 & 0.451 & 0.637 \\
\hline 20 & $425 \pm 10.0$ & $298 \pm 12 \cdot 2$ & -3 & \pm 8.4 & 0.460 & 0.632 \\
\hline
\end{tabular}

$\dagger$ Standard deviation of mean. 
BALANCE STUDIES WITH STOCHASTIC INPUTS

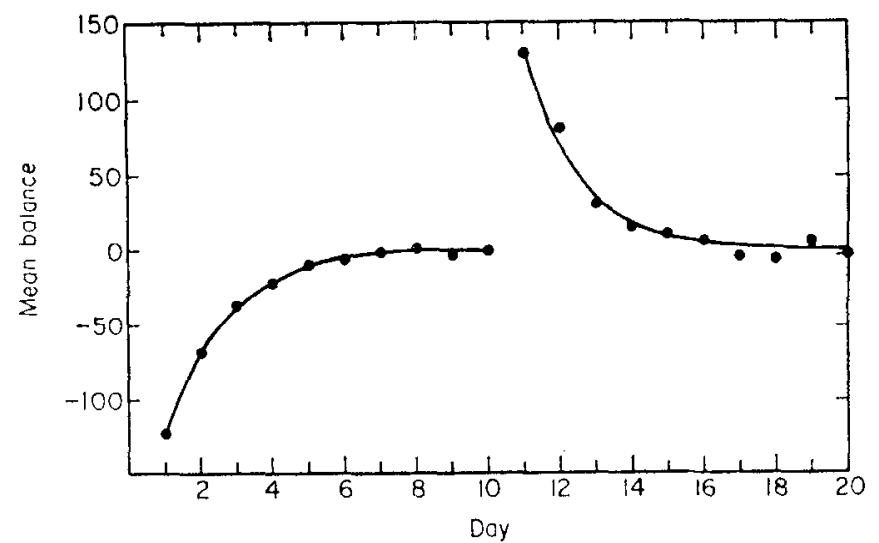

FIG. 5. Graph of sample mean balance for transition periods after step changes in distribution of intake between a low intake (days 1 to 10) and a high intake (days 11 to 20 ). Sample size for each day is 100 .

TABLE 3

Summary of regression of balances against intake by days, during transition period after step changes in distributions of intake

\begin{tabular}{ccccc}
\hline Day & Intercept & $\begin{array}{c}\text { Standard } \\
\text { deviation of }\end{array}$ & $\begin{array}{c}\text { Slope } \\
\text { A }\end{array}$ & $\begin{array}{c}\text { Standard } \\
\text { deviation of }\end{array}$ \\
\hline 1 & -198.6 & 16.2 & 0.72 & B \\
\hline 2 & -118.0 & 10.1 & 0.48 & 0.15 \\
3 & -95.7 & 6.5 & 0.56 & 0.06 \\
4 & -91.4 & 5.6 & 0.68 & 0.05 \\
5 & -86.9 & 4.1 & 0.73 & 0.03 \\
6 & -72.2 & 3.2 & 0.63 & 0.03 \\
7 & -65.9 & 4.8 & 0.58 & 0.04 \\
8 & -77.8 & 4.6 & 0.69 & 0.04 \\
9 & -78.9 & 4.3 & 0.70 & 0.04 \\
10 & -71.7 & 3.9 & 0.65 & 0.03 \\
11 & -68.1 & 3.2 & 0.63 & 0.01 \\
12 & -143.3 & 10.8 & 0.69 & 0.03 \\
13 & -149.9 & 11.7 & 0.59 & 0.04 \\
14 & -177.7 & 13.3 & 0.63 & 0.04 \\
15 & -203.8 & 11.5 & 0.70 & 0.03 \\
16 & -191.6 & 10.7 & 0.65 & 0.03 \\
17 & -210.5 & 12.8 & 0.69 & 0.04 \\
18 & -194.0 & 11.7 & 0.64 & 0.04 \\
19 & -189.4 & 9.8 & 0.64 & 0.03 \\
20 & -176.8 & 12.0 & 0.58 & 0.04 \\
\hline
\end{tabular}


low intake period and $306 \cdot 1$ for the high intake period. Table 2 summarizes the results for the 20-day cycle. Note that the first day on the low intake is the first day on low intake after transition from the high intake. Figure 5 presents a plot of the mean daily balances for the 20-day cycle. Table 3 gives the regression lines of daily balance on intake obtained by the standard least squares fitting method. The application of an analysis of covariance to the data gave no significant differences between the slopes for the different days at the $P=0.001$ level but did give a significant difference between the intercepts at the $P=0.001$ level. This was, of course, to be expectedequation (29).

This work was supported in part by grant 5T1 GM892 from the National Institute of Health.

\section{REFERENCES}

ARDEN, B. W. (1962). "An Introduction to Digital Computing". Reading, Massachusetts: Addison-Wesley Publishing Co., Inc.

GAlleR, B. (1962). "The Language of Computers". New York: MeGraw-Hill Book Co. Rụstagi, J. S. (1964). Archs envir. Hlih, 8, 68.

Vovart, R. L. \& London, W. T. (1964a). Am. J. clin. Nutr. 15, 124.

Vovaht, R. L. \& LoNDON, W. T. (1964b), Am. J. clin. Nutr. 14, 186. 